\title{
EVALUACIÓN DE LA ACCIÓN PEDAGÓGICA DESDE EL ESCENARIO TRANSVERSAL DE SABERES EN VENEZUELA
}

Romay Zuleima Flórez Santiago ${ }^{[1]}$

\section{Resumen}

La evaluación del desempeño docente desde el escenario universitario venezolano, se refleja en un horizonte crítico, reflexivo y epistemológico-filosófico representado por autores como Bolívar, Lanz, Kemmis, entre otros, quienes a través de sus voces sobre la transversalidad, la evaluación del trabajo docente, el trabajo en equipo, la complejidad y el currículum, generan otra visión de la valoración de la educación a nivel universitario con miras a la formación del ciudadano crítico que expresa sus ideas de forma escrita u oral. Considerando estos autores y la actual antítesis sobre universidades mercantilistas, planteamos la creación de una base teórica crítica-reflexiva que argumenta diferentes visiones, las cuales reflejan un entramado intersubjetivo subyacente en el proceso de evaluación del rol docente, su desempeño educativo y el egresado universitario. Para ello, a través de procedimientos metodológicos hermenéuticos se ha decidido observar, interpretar, analizar, reflexionar y dialogar con docentes y estudiantes sobre este fenómeno complejo de la evaluación del docente y el desarrollo de la creatividad en la producción de ideas por parte del estudiante, generando un enfoque crítico-reflexivo en cuanto a una actual visión del desempeño docente a nivel universitario, que contribuya con una formación de estudiantes críticos transformadores de sociedades.

Palabras clave: evaluación; acción pedagógica universitaria; egresados críticotransformadores.

\section{Summary}

The evaluation of teacher's performance from the Venezuelan university scene is reflected in a critical, reflexive and philosophical-epistemological horizon represented by authors such as Bolivar, Lanz, Kemmis, among others, who through their voices on transversality, teacher's evaluation, the teamwork, the complexity and the curriculum, created another view regarding the university education evaluation, that involves the training of critical citizens whom expresses their ideas in written or oral form. Considering these authors and the current antithesis of mercantilist universities, the creation of a critical-reflexive theoretical basis was proposed that can argue different

[1] Dra. en Educación. Docente adscrita al Departamento de Socio-Humanidades de la Universidad de Oriente Núcleo Nueva Esparta, Venezuela. Correo: romayflorez@hotmail.com 


\section{EDUCACIÓN}

views, which reflect an underlying intersubjective framework for the evaluation of the teaching process, their educational performance as well as the graduates. To do this, it's necessary to use hermeneutical-methodological procedures that will permit us to observe, interpret, analyze, reflect and dialogue with teachers and students about this complex phenomenon of teacher's evaluation and development of students creativity in the production of ideas, generating a critical and reflexive approach to the current view of a university teacher's performance, which contributes to training students with a critical view to transform societies.

Keywords: Evaluation; university pedagogical action; critical-transformers graduates.

\section{Introducción}

El contexto social contemporáneo requiere de ciudadanos integrales y críticos que formen parte activa dentro de las transformaciones vertiginosas de esta época y la formación de este tipo de ciudadanos es posible considerando elementos como la evaluación del proceso educativo, del currículo universitario, la universidad y los docentes. Todos estos elementos son clave para una educación integral del estudiante que desarrolla la capacidad crítica y el compromiso a ser parte activa en los cambios socio-culturales emergentes.

Del mismo modo, esta educación integral debe estar enmarcada en principios éticos que permitan la formación de un estudiante comprometido con la sociedad, crítico y transformador. Un estudiante que se enmarca en un perfil como el expuesto por Bolívar (2000), comprometido, activo para mejorar continuamente, que coordina en grupo, que se esfuerza por compartir metas en colaboración, desarrolla perspectivas comunes, entre otras características. Para ello, la universidad debe asumir el cambio configurándose como un espacio transversal de saberes donde la educación se fundamente en la responsabilidad, la ética, en el cumplimiento de deberes y derechos propios y colectivos de quienes forman parte de este sistema. Esto es posible, si los docentes estamos conscientes de los cambios que se suscitan en nuestro entorno, es decir, si conocemos nuestras realidades sociales y nos comprometemos a cambiar de la pedagogía tradicional depositaria hacia una más crítica y participativa que se adecúe mucho más a estas realidades contemporáneas.

No obstante, en diversas universidades venezolanas, existen numerosos docentes que no se han comprometido con el actual clima educativo, social y cultural, continuando éstos con sus ejercicios docentes tradicionalistas en el que ni los estudiantes ni los docentes toman parte activa en los proceso de cambio. De allí, la importancia de la elaboración de este trabajo de investigación, en el cual se consideró mi experiencia vivida como estudiante de pre-grado, post-grado y doctorado en tres universidades 
diferentes de Venezuela, así como también, se llevó a cabo una investigación documental, cualitativa, hermenéutica, con el propósito de organizar, interpretar, comprender y relacionar la información teórica que permita la elaboración de nuevas perspectivas conceptuales o argumentativas como herramientas para el desarrollo de formas actualizadas del proceso de enseñanza y aprendizaje que vaya acorde con las tendencias socio-culturales contemporáneas.

El resultado de esta investigación refleja una base teórica de gran utilidad para quienes formamos parte protagónica en la universidad, entendida en este trabajo como el escenario transversal de saberes. Considerando lo expuesto, el presente trabajo se estructura con un marco conceptual en el que se refleja: La visión de la universidad venezolana en los tiempos actuales, el currículo universitario venezolano actual y el currículo con miras a la universidad transformadora, encontramos aportes teóricos relacionados con el desempeño docente y la evaluación de la educación a nivel universitario. Del mismo modo, un marco metodológico: fundamentado en la interpretación y análisis de estas teorías. Finalmente, se presentan resultados de la investigación, conclusiones y las referencias bibliográficas.

\section{Marco conceptual}

\section{La universidad contemporánea venezolana como espacio transversal de saberes}

Somos parte de un recinto donde se destaca nuestra noble labor como docentes. Un recinto que alberga una diversidad de seres con sueños, ilusiones, esperanzas y que recurren a nosotros en búsqueda de apoyo y orientación. Con esto se espera que sea llevada a cabo nuestra vocación, con amor, sin fingimiento, eliminando la discriminación y adhiriéndonos al buen trato hacia los otros. Un recinto que se mueve dentro de un sistema globalizado y que a su vez, en palabras de Huget (2004), posee un papel fundamental ante lo que llamamos la sociedad del conocimiento. Este autor expresa que la educación superior, se encuentra frente a un reto ineludible en el cual debe "adoptar estándares de calidad educativa internacionales para poder ser competitivos, sin abandonar nuestros propios estándares de calidad desarrollados a lo largo de años de experiencia académica" (p. 2). Considerando esta cita, no refiero lo competitivo al campo laboral sino al logro de competencias que nos destaquen como entes sociales axiológicos y epistemológicamente formados en los predios universitarios para enfrentar críticamente cualquier cambio que requiera la sociedad.

Se trata de un giro humanístico que presupone la existencia globalizadora pero que forja el trabajo en grupo, trabajo que desde tiempos remotos ha debido su existencia a la cohesión social para la productividad, pero que en los actuales momentos, sea este trabajo constituido para las transformaciones que impliquen siempre un bien común. Al hablar de transformaciones reclamadas por nuestros entornos, manifestamos que 


\section{EDUCACIÓN}

en los diversos estratos sociales, el detrimento de las valoraciones, las evaluaciones o la reflexión, en cuanto a las actitudes humanas, ha cobrado fuerza. Ejemplos de ello, lo vemos en el deterioro ecológico, las divisiones y falta de valores en los núcleos familiares, la falta de ética profesional, la influencia de otras culturas logrando el desplazamiento de la nuestra, entre otros.

El aporte de Lanz y Fergusson (2005), nos habla sobre el compromiso y la evaluación del sistema educativo en general los cuales deben proyectarse en la generación de ciudadanos, docentes y la sociedad que actualmente se requieren. El aporte de estos autores está fundamentado en el diagnóstico calificativo de las realidades socioemergentes y sobre la base de los resultados arrojados, la generación de una apertura al trabajo cooperativo, como todo un cuerpo, bien definido y unificado entre sí por todas sus coyunturas que se apoyan y se ayudan mutuamente, según la actividad o disciplina propia de cada miembro desarrollando un crecimiento que se edifica en amor como el Supremo lo presenta, al bienestar de la comunidad que se integra y que participa, democrática, libre y soberanamente. Siguiendo este mismo orden de ideas, en los diferentes entornos que conforman nuestras sociedades, las características fundamentales que se destaquen deben relacionarse con el factor reflexivo-evaluativo, armónico, el diálogo y del punto de vista crítico surgido de la capacidad autónoma de producir conocimiento transdisciplinariamente; para ello, la universidad, los docentes y el sistema educativo deben enmarcarse en dichas características.

$\mathrm{Al}$ respecto, Lanz y Fergusson (2005) proyectan:

La doble función paradójica de la universidad: adaptarse a la modernidad científica e integrarla, responder a las necesidades fundamentales de formación, ofrecer profesorado para las nuevas profesiones, pero también, y sobre todo, transformar el pensamiento que la piensa, repensar la modernidad y ofrecer una enseñanza transprofesional, transdiciplinaria, transtécnica, es decir una nueva cultura. (p. 95).

Esta cita hace referencia a una incongruencia existente en las universidades venezolanas en relación a la perspectiva crítica reflexiva, ya que la formación modernocientífica que cubra necesidades inmediatas y la integración de los procedimientos puramente científicos modernos, excluyen procedimientos a nivel universitario que a través de sistemas evaluativos comprendan o elaboren un currículo que se adapte a los contextos y a las bases que representen evolución y desarrollo constante y que se constituya de fundamentos epistemológicos que apuesten a lo cualitativo, a lo holístico a las complejidades multi y transdisciplinarias y a las participaciones activas de los protagonistas que conforman el marco universitario.

Para esto último, es menester que dentro de las funciones universitarias se lleven a cabo encuentros de saberes significativos en los que se expongan el valor del entorno entretejido con realidades que partan desde lo general a lo particular; esto es, 
considerar fenómenos subyacentes en el ámbito educativo en general, las políticas educativas, las evaluaciones y participaciones dentro de las aulas de clase, así como también, de lo que actualmente vive el mundo y se vive en nuestras propias localidades o espacios geográficos y viceversa, motivados por un espíritu y necesidad de descubrimiento que permitan ideas críticas transformadoras.

Sobre lo expuesto, Lanz y Fergusson (2005) agregan:

Se trata pues, de comprometer nuestro esfuerzo en promover cambios radicales en la política académica universitaria, pues hemos comenzado a entender que, de lo contrario, la educación que impartimos continuará reforzando la pedagogía de la domesticación, alimentando una visión instrumental de la naturaleza y el mundo, y reproduciendo un modo de vida cuya lógica de la rentabilidad económica, subordina el interés colectivo por el mejoramiento de la calidad de la vida humana y la salud mental a los intereses individuales o de los grupos económicos y políticos que controlan el poder. (p. 97).

Esto se refiere a que los yacimientos y esquemas que configuran una universidad con características tradicionales se están viendo removidos por nuevas visiones fundamentadas en lo colectivo, en el voluntariado, en la otredad, la empatía, la hermenéutica de realidades y la dialéctica de transformación. Esto es entonces, un llamamiento a las universidades para que evalúen lo emergente y den un giro que apunte a nuevas discusiones y acuerdos de transformación curricular.

\section{Currículo universitario}

Sobre la base de lo expuesto, la hermenéutica de realidades y la dialéctica transformadora dentro del currículo universitario, se caracterizan por ser procedimientos filosóficos en los que aparecen el mundo complejo de la comprensión, las relaciones entre el sujeto, el objeto, los contextos particulares de ellos para luego críticamente generar un nuevo conocimiento que contribuya a transformar esas realidades emergentes. $\mathrm{Al}$ respecto, tenemos el aporte de Gadamer, (1998) quien explica que "la hermenéutica describe todo el dominio del entendimiento entre los hombres" (p. 231); esto quiere decir, comprendernos entre nosotros, el género humano y todo cuanto nos rodea, todo lo relacionado a nuestros contextos sociohistóricos, con miras a soluciones de situaciones que nos favorezcan en conjunto. Ante lo expuesto, nos referimos a la evaluación y emancipación de un currículo fundamentado en teorías críticas que lo configuren en un marco dialéctico como el descrito por Kemmis, (1993), quien nos plantea que: 
En vez de considerar los problemas de la sociedad como problemas de un agregado de individuos únicamente, o los problemas individuales como procedentes tan sólo de la determinación social de las vidas de los sujetos, el razonamiento dialéctico intenta desenredar las interrelaciones dinámicas, interactivas mutuamente constitutivas entre la vida del individuo y la vida social. Asimismo, el razonamiento dialéctico trata de entender las relaciones dinámicas, interactivas, mutuamente constitutivas entre la teoría y la práctica, considerando a ambas socialmente construidas e históricamente desarrolladas, más que como si cada una determinara en exclusiva a la otra. (p. 83)

Este planteamiento dialéctico refiere a un carácter mediador en la constitución formativa del individuo que interactúa con los otros, que depende de un contexto socio-histórico y no desvirtúa la teoría de la práctica en el día a día. La evaluación del proceso educativo bajo el razonamiento dialéctico, enmarcado en la noción de la unidad de opuestos considerando las contradicciones y generando transformaciones, es fundamental para tener otra visión del currículo universitario.

Estamos ante transformaciones curriculares que versen hacia una autonomía con libertades en razonamientos que emancipen a los protagonistas de los recintos del saber de las ideas falsas, del distorsionamiento comunicativo y de la mal interpretación de la relación social que constriñen las acciones humanas y las sociales.

Todo esto representan presupuestos, que desde la dialéctica, nos incursionan en el campo curricular inmerso en la educación, la universidad, la sociedad y el proceso evaluativo que los envuelve. Una posición crítica, ante la trayectoria que en su momento desarrollaron las teorías técnicas y prácticas de los currículos, se fundamentó en la cientificidad o carácter crítico creativo de los que deberían estar constituidos actualmente los currículos de educación.

Currículos que invitan, según Kemmis (1993) “a los estudiantes a pensar por sí mismos y no a repetir los pensamientos de sus profesores” (p. 75). Considerando este argumento, se puede decir en pocas palabras que el estudiante aborde las ideas comprendiendo el discurso que le quieren expresar sus iguales, enfoque esa idea o el tema desde varias perspectivas, analice la fuente de información o el tipo de texto en el que está inmerso el discurso, decida cuál texto o idea es relevante para su posterior construcción de ideas, realice una comprensión global de los mismos y finalmente construya una visión crítica de los discursos inmersos en cualquier tipo de texto con el propósito de ser partícipe en cualquier cambio que requiera su entorno.

\section{Currículo por competencias}

En continuidad con lo anteriormente expuesto, sabemos que los antiguos esquemas en el campo educativo se desvanecen por la actual crisis de saberes teóricos los cuales 
se ven abordados por los ascendentes y dinámicos contextos sociales; es menester entonces, diagnosticar para la creación de nuevas construcciones epistemológicas y así, comprender mejor las situaciones que se presenten. El aprendizaje de competencias deben cumplir con el requerimiento social, cuya formación del individuo se fundamente en el desarrollo de conocimientos para toda la vida y en su permanente actualización del saber.

Si consideramos las actuales evaluaciones del sistema educativo universitario, dentro de los cambios que recientemente amerita la universidad, se plantea la necesidad de reflexionar sobre el diseño de un currículo orientado por el enfoque de competencias pero, que quede claro que son el tipo de competencias más humanas y no mercantilistas. Este enfoque no figura simplemente una mentalidad instrumental, técnica, cuantitativa de producto sino que propicia y estimula una reflexión sobre la elaboración de un nuevo diseño curricular que vaya más a lo integral; es decir, más al aprender a pensar a ser, hacer, conocer y convivir.

Esta visión integral configura a un estudiante que en cualquier faceta o situación cotidiana, aprende a observar todo cuanto le rodea, aprende a pensar, a interpretar las situaciones socioculturales que le rodean, a relacionar estas situaciones con la experiencia en la universidad de manera que su aprendizaje sea significativo, que desarrolle el hábito de la lectura, genere preguntas constantemente y trate de responderlas a través de la interacción con los textos que lee y del diálogo que establezca con quienes le rodea.

El tipo de competencias integrales adaptadas a la propuesta hecha por Lanz y Fergusson (2005) nos muestra a un ser cuyas propiedades lo configuran como crítico, creativo, analítico, espontáneo, libre, sensible, curioso y lleno de un espíritu cuestionador y de cuestionamientos constantes; con pertinencia cognitiva y cognoscitiva, esto es, que conoce porque evalúa, investiga, comprende, infiere y construye saberes de forma generalizada e interactúa.

Otro enfoque sobre competencias, lo podemos encontrar en el aporte de Aristimuño (s.f.) quien las define como el conjunto de atributos relacionados con los conocimientos, los valores, la ética, la disciplina, habilidades, actitudes y responsabilidades desarrolladas por una persona durante su proceso de formación o como producto de la misma.

Son competencias inmersas en la complejidad que conforma al ser con su entorno y demuestran el óptimo desenvolvimiento profesional con respecto a este ser en sí, sus ideas y a las tareas contextualizadas que desempeña. El óptimo desenvolvimiento profesional lo podríamos incluir en el dominio de un área determinada del conocimiento. El aporte de Barnett, (1992) con respecto a este dominio, habla de personas que en las diversas disciplinas de conocimientos, como la química, filosofía, psicología, historia e idiomas, logran la comodidad y el adecuado desenvolvimiento; ya sea en laboratorios, 
en el desarrollo de un argumento válido sobre una determinada situación, manejo de estadísticas apropiadamente, capacidad de localizar y analizar material histórico y la destreza de hablar otro idioma para efectos de comunicación.

La universidad, a través de un currículo por competencias, debe asumir el compromiso, como lo exponen Lanz y Fergusson (2005) de presentar egresados que se hagan cargo de la complejidad de los cambios que actualmente se viven, que sean críticos y dialoguen datos de una realidad que desde hace tiempo, viene presentándose como crisis que amerita reformas para confrontarla; en términos específicos, esto es evaluación de la acción pedagógica. Sobre esta base medular es que los esfuerzos de ajustes, realizados por los protagonistas inmersos en el sistema universitario, deberían encaminarse a planes de estudios que fortalezcan las competencias de tipo humanística.

La configuración del futuro egresado y el repensar de una evaluación y transformación curricular universitaria, enfocada en el horizonte humanístico, representa la conformación de un sistema complejo que abarca aspectos relacionados con nuevas estrategias educativas, contenidos, objetivos, el diario convivir educativo, programaciones y evaluaciones. Este último lo consideramos como uno de los aspectos más importantes, ya que de ello surge el reconocimiento del logro de objetivos o metas enmarcadas en los cambios positivos que hemos venido argumentando.

Las competencias que aquí se proponen no aplican a evaluaciones de producto porque ellas son inherentes a procesos; es decir, procesos encaminados hacia nuevos horizontes que reflejan un aprender a convivir con las diferencias y que requieren de mucho más observación y mucha más dialogicidad que permita los acercamientos y las comprensiones entre los protagonistas inmersos en el proceso educativo y el acuerdo mutuo para lograr ver lo que va bien y lo que podría mejorarse para la producción de conocimientos desarrollados por los mismos.

\section{Evaluación de la acción pedagógica a nivel universitario}

Partiendo de una cosmovisión evaluativa podemos decir que la universidad cumple un papel preponderante en la evolución de cualquier realidad a través del conocimiento y las aplicaciones productivas que de ella emergen y que se fundamentan en un sistema complejo multidimensional, no reduccionista, de carácter político, económico, educativo, social y cultural. Estamos conscientes de la magnitud del rol de la universidad y de la complejidad del conocimiento que debería implementarse en la misma; así como también, que un sólo docente no tiene la solución o salida a lo expuesto; sin embargo, estamos conscientes de ese todo que debemos asumir y enfrentar, considerando también las partes y es en el aula de clase por donde debemos comenzar. De la multi-demensionalidad universitaria, consideremos un aspecto de la misma y observemos cómo podríamos de algún modo formar parte activa de los cambios requeridos en los actuales momentos. 
Esta perspectiva de construcción social integrada, participativa, organizativa y de formación, guarda relación con la concepción que Bolívar (2000) hace sobre las "Organizaciones que Aprenden" cuyos principios se fundamentan en diversas literaturas necesarias para generar aprendizajes dentro de esas organizaciones; según este autor, esos principios destacan

compromiso activo por la mejora continua, grupos coordinados que se esfuerzan por compartir metas en colaboración, desarrollo de visiones comunes sobre los objetivos de la organización, difusión de las mejores prácticas en toda la organización, aprendizaje y desarrollo profesional del personal, examinar críticamente las prácticas habituales, experimentar nuevas prácticas, disposición para cambiar los marcos habituales de pensar, desarrollar un espíritu de empresa comprometida con la mejora, redes horizontales de flujo de información internamente y con el entorno exterior, habilidades para comprender, emplear sistemas dinámicos de funcionamiento, entre otros. (p. 24)

Estos principios configuran la universidad como un escenario transversal de saberes, en el que diversas disciplinas y grupos disciplinarios convergen y se interrelacionan en la búsqueda de generación de nuevos conocimientos. Así mismo, la configuran como un ente complejo, consciente de los cambios actuales y de que está ante un reto por dichos cambios. La universidad bajo estos principios posee una sensibilización hacia lo social, conociendo, haciendo, siendo y conviviendo, apartando la discriminación y los abusos de poder, ayudando a desarrollar destrezas y habilidades para la construcción de conocimientos. De la misma manera, cumplir con esos principios es forjar actitudes morales, de responsabilidad, respeto a la diversidad y todo lo expuesto, sobre la base del trabajo cooperativo y el diálogo dentro del aula de clase.

Esto quiere decir, el aula de clase como organización, esto es una visión bastante comprometedora y debemos estar conscientes que adoptar posiciones críticas de aprendizaje como organización, en un ambiente con un fluir práctico y colaborativo, también, por naturaleza humana, trae consigo un cúmulo de disensos o conflictos sobre todo por la impactante influencia de carácter socio-político existente en el entorno. No obstante, apostamos a un despliegue comunicativo de políticas y de acercamiento por parte de los docentes y los estudiantes que enaltezcan la universidad como un verdadero centro de organización que aprende a crearse su propia imagen como organismo de formación e innovación con un alto valor en los procesos de investigación y trabajo cooperativo.

Incluir el compromiso ante lo expuesto, en palabras de Bolívar (2000) refiere un lugar: 
Donde una mayor autonomía pudiera incrementar la profesionalidad de los profesores y su implicación con la mejora, mediante formas comunitarias de participación y colaboración en el centro escolar, que provoquen una articulación horizontal, en una concepción “orgánica” del centro escolar. Generar progresivamente una implicación y compromiso en torno a una agenda de actividades en el trabajo cotidiano, expresión de un conjunto de valores compartidos, proporciona un contexto propicio para la innovación educativa, al tiempo que posibilita la organización como conjunto que aprende. (p. 208)

Esto se refiere a una visión de cambio hacia un tipo de organización sistémico y requiere de constante auto-evaluación por parte de los docentes y estudiantes, con el propósito de buscar siempre las mejoras a través de un trabajo en conjunto y partiendo de sus experiencias pasadas como referencias de lo que se debió o no hacer y proyectarse a futuro con nuevas perspectivas y giros cualificadores que garanticen soluciones creativas y transformadoras internas y externas.

Bajo esa égida de aprendizaje organizativo y todo lo que éste implica para cumplir metas que vayan de lo individual a lo grupal y viceversa, se enmarcan las consideraciones y distinciones que Bolívar (2000) señala con respecto a los aprendizajes tácitos y explícitos; Para este autor, "una organización que se "mueve" crea conocimientos mediante continuos procesos de comunicación y transferencia entre los miembros, de un saber tácito al explícito y viceversa" (p. 156). Extrapolemos lo expuesto al quehacer diario de los docentes y los estudiantes, quienes forman parte importante en ese conjunto participativo dentro de la organización universitaria que aprende.

El caso del tratamiento de la evaluación en los planteamientos que argumentan la transformación o desconstrucción de antiguos esquemas inherentes a este proceso, al acto pedagógico, en la formación del egresado crítico, el docente comprometido con esa filosofía crítico-reflexiva y el entorno universitario, la influencia de este escenario transversal de saberes, es absolutamente predominante. La transversalidad antes expuesta hace referencia a un sistema que involucra a una serie de aspectos que compactan seres integrales y que bajo un enfoque de diálogo, hacen la plataforma o crean un espacio abierto en donde se da cuenta una formación integral donde la producción de conocimientos cubre un espacio bien establecido desde el aula de clase.

La evaluación siempre ha sido tema de debates y de supuestos que defienden la re-conceptualización de la misma debido a los cambios demandados por las sociedades. La evaluación debe cobrar vida allí, en las prácticas académicas, en el papel de las bibliotecas, en el día a día del docente, en el campo tecnológico de la información y comunicación y en las actividades de extensión. Todo un sistema que proyecta una cosmovisión de la universidad cimentada en la constante búsqueda de la calidad educativa. 
En términos generales, evaluar la acción pedagógica es desarrollar una política evaluativa de la educación y pasar del contexto mercantilista; de empleo-universidadprofesión; de formadores de seres puramente de conocimientos; al contexto de formación de un ser ideal y absolutamente preparado para enfrentar y resolver situaciones contextuales enmarcadas en lo cognoscitivo, en lo personal, profesional, espiritual, ético, social y mundial.

\section{El docente universitario}

En la construcción de saberes suscitados en el campo educativo, muchos son los factores que fluctúan y permiten el consenso cognoscitivo. En este proceso, permanecerá la figura brillante, sublime y latente del docente, a decir, ¿Quiénes sino nosotros los forjadores atentos y serviciales para que tan anhelada tarea de construir conocimientos pueda cumplirse? Nosotros los docentes por naturaleza misma tenemos la noble labor de guiar, debemos conocer diferentes senderos que nos lleven a todos, estudiantesdocentes, a la cumbre del saber $y$, ser conocedores de esos senderos implica un espíritu de búsqueda, un espíritu inmanente a lo desconocido y a la curiosidad.

En realidad estamos ante un tema de reflexión, de toma de consciencia y de desarrollar nuevas actitudes. Debemos comenzar, en tanto, a formar a quienes a futuro serán formadores del ciudadano social. Si nos revestimos de ese alto porcentaje de conciencia de valores habremos hecho esa introspección y lograríamos transformarnos y redescubrir nuestro otro "Yo". Transformaciones hacia un nuevo docente que se apuesta a los cambios, los asume con integridad desde la complejidad de una nueva constitución y desarrolla nuevas actitudes, axiológicas, epistemológicas y pedagógicas.

La visión pedagógica matizando las actitudes antes expuestas requiere de posturas críticas que reorientan el perfil del educador en un marco de teorías filosóficas que justifican las tendencias a unas prácticas socio-educativas en las que se llevan a cabo análisis de realidades existentes. El educador crítico puede generar un ámbito pedagógico-didáctico enaltecido por aspectos fundamentales comunicativos y en el que las situaciones inherentes de conflictividad deben ser aprovechadas atendiendo rasgos lingüísticos conducidos bajo entornos organizativos permitiendo la participación y la creación de nuevos discursos que involucren al sujeto con su entorno. Estamos entonces ante un docente universitario no dualista relacionado con una didáctica ecológica y comunicativa como la descrita por Rodríguez (1997) la cual, hace referencia a un modelo de carácter ecológico-comunicativo que se corresponde con la teoría de acción comunicativa:

Es el modelo que, según mi opinión, puede representar a la teoría crítica, ya que el planteamiento ecológico, abre un sin fin de comunicaciones con los sistemas exteriores al aula y al mundo de la vida, donde habitan los grandes protagonistas del proceso de enseñanza y aprendizaje (p.145) 
Esto hace referencia al docente que convence mucho más con sus acciones que con sus palabras y conocimientos. Un docente guía cuyo comportamientos refleje un alto valor ético-moral, un docente que se conozca por ser observador de realidades, ávidos de conocimiento, investigadores, hermenéuticos, mayéuticos, participativos en las transformaciones estructurales, coyunturales de la universidad, de los entornos y forjadores de esos frutos convertidos en futuros egresados universitarios encaminados hacia el conocimiento, hacia la cultura y hacia la sociedad.

Esto es una tarea ardua y para algunos quizás quimérica. De allí que surge la siguiente interrogante: $¿$ Está el docente universitario dispuesto a aceptar los cambios que se ameritan para enfrentar la realidad y prepararse para re-orientarla? En algunas oportunidades, en el espacio universitario en el que se forman futuros formadores es decir, en las escuelas de educación, se presenta una situación que de alguna manera, responde a la interrogante realizada. Esta situación es que en la mayoría de los casos por naturaleza humana, todo cambio implica un rechazo.

\section{Resultados}

A través de un estudio de carácter interpretativo-hermenéutico sobre la base de experiencias como estudiante y docente en diversas universidades, así como también por el análisis de diversas teorías del campo educativo, se obtiene como resultado este trabajo de investigación ensayístico crítico-reflexivo en cuanto a una actual visión del desempeño docente en algunas Universidades Venezolanas que pueda contribuir con una formación de estudiantes críticos transformadores egresados de universidades con currículos que se fundamenten en las competencias y así formar parte activa de la dinámica social emergente.

\section{Conclusiones}

En los actuales momentos la necesidad de conocimiento por parte del ser humano ha sido clave en el contexto filosófico y epistemológico, esta necesidad se ha incrementado y ha sido fuente inspiradora de los cambios sociales y culturales contemporáneos inmersos en una incertidumbre y complejidad propias de esta época. En este sentido, se hace prominente un ser social, un docente y una universidad críticos generadores de ideas que forme parte activa de los vertiginosos y complejos cambios que requieren la consolidación de nuevos conocimientos que influyan positivamente en esos cambios y que permitan una sociedad más humanizada. Por estas razones, las universidades deben consolidar proyectos que promuevan un sistema educativo basado en fundamentos de teorías críticas y de la complejidad, a fin de configurar egresados críticos generadores de ideas e inmerso en este sistema universitario se encuentra la participación del docente que se comprometa con dichos cambios. De allí que las prácticas pedagógicas se tornen más a lo creativo que a la tradicional perspectiva pasiva depositaria. 
Dentro de esas prácticas pedagógicas, la filosofía y elementos como la hermenéutica y la dialéctica conforman un entramado en el que el currículo universitario que cobra fuerza dentro del proceso de transformación y configuración de un estudiante crítico-reflexivo generador de ideas. En este sentido, la universidad venezolana como escenario transversal de saberes debe asumir y proyectar una educación al nivel de la complejidad que conforme la sociedad.

\section{Lista de referencias}

Aristimuño, A. (s/f.) Las competencias en la educación superior: ¿demonio u oportunidad? Obtenida en Febrero, 16, 2008 de www.unizar.es/ice/rec-info/Curso45/.pdf

Barnett, R. (1992). Los límites de la competencia. El conocimiento, la educación superior y la sociedad. Obtenida en Febrero, 16, 2008 de http://www.educar.org/MFDTIC/ Documentos/perfildocente.asp.

Bolívar, A. (2000). Las organizaciones que aprenden. La Muralla. Madrid.

Gadamer, H.G. (1998). La hermenéutica tras la huella. Obtenida en Junio, 22, 2012 de http://digitooluam.greendata.es//exlibris/dtl/d3_1/apache_media/l2v4bglicmlzl2robc9km18xl2fwywnozvgtzwrpys8ymje4oq.pdf .

Huget, A. (2004). Apuntes acerca de la evaluación educativa. Obtenida en Febrero, 16, 2007 en http://www.cosdac.sems.gob.mx/descarga_archivo. $\mathrm{php} ? \mathrm{~b}=3 \&$ documento $=$ doc_29.pdf .

Kemmis, S. (1993). Hacia la teoría crítica del currículum. El currículum: más allá de la teoría de la reproducción. Morata. Madrid.

Lanz, R. y Fergusson, A. (2005). La reforma universitaria en el contexto de la mundialización del conocimiento. Obtenida en Mayo, 11, 2007 de http://www.cosdac.sems. gob.mx/descarga_archivo.php?b=3\&documento=doc_29.pdf .

República Bolivariana de Venezuela. Ministerio de Educación Superior. (2003). Propuestas para la discusión de Educación Superior. Caracas zo de abril.

Rodríguez, R. M. (1997). Hacia una didáctica critica. Madrid. La Muralla.

Tavárez, M. (2005). Perfil del docente latinoamericano: ¿mito o realidad? Obtenida en Febrero, 16, 2008 en http://www.educar.org/MFDTIC/Documentos/perfildocente.asp

Zemelman, H. (2008). Concepción del conocimiento y conciencia histórica en el quehacer universitario contemporáneo. Conferencia Inaugural presentada en el II Congreso Internacional de Educación Superior, Cumaná. Venezuela 